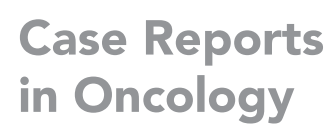

\title{
Hypercalcemia Associated with Extramammary Paget's Disease
}

\author{
Tetsuko Sato $^{a}$ Yoshihisa Wada ${ }^{b}$ Nobuhiko Kamitanic \\ Takenobu Yamamoto ${ }^{a}$ Yumi Aoyama ${ }^{a}$ Wataru Fujimoto ${ }^{a}$ Ryo Tanaka ${ }^{a}$ \\ aDepartment of Dermatology, Kawasaki Medical School, Kurashiki, Japan; \\ ${ }^{b}$ Department of Nephrology/Hypertension, Kawasaki Medical School, Kurashiki, Japan; \\ 'Department of Radiation Oncology, Kawasaki Medical School, Kurashiki, Japan
}

\section{Keywords}

Behavioral disturbance $\cdot$ Extramammary Paget's disease $\cdot$ Hypercalcemia $\cdot$ Parathyroid hormone-related protein

\begin{abstract}
Hypercalcemia of malignancy occurs in up to one third of patients at some point during the course of their advanced stage. The majority of them is caused by humoral hypercalcemia of malignancy due to systemic secretion of parathyroid hormone-related protein (PTHrP) by tumor cells. Extramammary Paget's disease is a slow-growing cutaneous malignancy commonly limited to the epidermis of the anogenital region, but rarely becomes invasive and metastatic to distant sites. Herein, we report a 70-year-old male patient with metastatic extramammary Paget's disease. He consulted our hospital with altered consciousness and tumor in his genital area. Physical examination revealed erythematous plaque with a tumor on the scrotum and perineum. It was diagnosed as extramammary Paget's disease (multiple liver metastases and multiple lymph node metastases by skin biopsy and image examination). Increases in serum-corrected calcium and PTHrP-intact levels $(15.3 \mathrm{mg} / \mathrm{dL}$ and $66.1 \mathrm{pg} / \mathrm{L}$, respectively) were confirmed. PTHrP immunohistochemistry showed positive staining in the tumor cells. We diagnosed humoral hypercalcemia of malignancy. We treated hypercalcemia with saline, furosemide, zoledronic acid, and elcatonin. Regarding the local control of the tumor, $30 \mathrm{~Gy} / 10 \mathrm{Fr}$ electron beam therapy was performed. However, treatment with zoledronic acid was only temporally effective to correct hypercalcemia, and an increased serum calcium level developed again. Concurrently, the liver metastases were rapidly enlarged, and his general condition gradually deteriorated. The patient died on day 55 . When patients with extramammary Paget's disease show unconsciousness, serum calcium level should be measured and PTHrP-producing tumor distinguished.




\section{Introduction}

Hypercalcemia has been reported to occur in $20-44 \%$ of patients with cancer [1-3]. Among them, $80 \%$ is caused by humoral hypercalcemia of malignancy (HHM) due to systemic secretion of parathyroid hormone-related protein (PTHrP) by tumor cells. HHM has been reported in squamous cell carcinoma, renal cancer, and ovarian cancer among others [1]. Although the symptoms of HHM are often mild and nonspecific, patients often develop neurocognitive disorders. The resulting metabolic derangements of HHM can be rapid and associated with poor prognosis.

Extramammary Paget's disease (EMPD) is a rare, slow-growing cutaneous adenocarcinoma that commonly occurs in sites rich in apocrine glands, such as the vulva. EMPD manifests as erythematous patches or plaques. Paget's cells are usually limited to the epidermis and epidermal appendages for a prolonged period of time. However, they rarely become invasive and infiltrate the dermis, or even become metastatic to the regional lymph nodes and distant sites. Several chemotherapy regimens to treat metastatic EMPD used up to date show limited effect and patients with distant metastasis exhibit a poor prognosis [4]. Hypercalcemia associated with EMPD is rare and has not been reported in English language literature.

\section{Case Report}

A 70-year-old man with a 6-month history of genital skin lesion presented with confusion. The patient's abnormal behavior of frequent call and polyuria developed 3 weeks ago. Eight days ago, he was admitted to a nearby hospital because of lumbago and difficulty in walking. The patient was referred to our dermatology unit for evaluation of the genital skin lesion. On inquiry, the patient was slowly replying and was disoriented. Physical examination revealed an eroded, erythematous plaque with a white-coated tumor on the scrotum and perineum (Fig. 1a).

Several enlarged inguinal nodes were readily palpable. The laboratory data on his first visit showed marked hypercalcemia: serum calcium $14.5 \mathrm{mg} / \mathrm{dL}$, corrected Ca $15.3 \mathrm{mg} / \mathrm{dL}$ (normal range, 8.8-10.1 mg/dL), with PTHrP-intact $66.1 \mathrm{pmol} / \mathrm{L}$ (normal, $<1.1 \mathrm{pmol} / \mathrm{L}$ ), PTH-whole $5.9 \mathrm{pg} / \mathrm{mL}$ (normal range, $8.3-38.7 \mathrm{pg} / \mathrm{mL}$ ), 1,25(OH) $)_{2}$ vitamin $\mathrm{D}_{3} 55.4 \mathrm{pg} / \mathrm{mL}$ (normal range, $20.0-60.0 \mathrm{pg} / \mathrm{mL}$ ), $25-0 \mathrm{H}$ vitamin $\mathrm{D}_{3} 14.3 \mathrm{ng} / \mathrm{mL}$ (normal range, 9.0-33.9 ng/ $\mathrm{mL}$ ), carcinoembryonic antigen $330 \mathrm{ng} / \mathrm{dL}$ (normal range, 0-5.0 ng/dL), and creatinine 1.63 $\mathrm{mg} / \mathrm{dL}$. A computerized brain scan revealed no occupying lesion suggestive of tumor or stroke. A computed tomography (CT) and magnetic resonance imaging (MRI) scan of the spine revealed a compression fracture of the 3rd lumbar vertebra, but not a pathological fracture indicative of metastasis. ${ }^{99 \mathrm{~m}} \mathrm{Tc}-\mathrm{HMDP}$ (hydroxymethylene diphosphonate) imaging and CT revealed no evidence of bone metastasis. Abdominal CT and echography revealed multiple metastases in the liver, and para-aortic, pelvic, and inguinal lymph nodes. Histopathology of a specimen taken from erythematous plaque showed a thickened epidermis occupied by neoplastic cells with large nuclei in the entire epidermis. Skin biopsy from the tumor showed infiltration of small, atypical cells arranged in nests and strands in the dermis (Fig. 1b). Atypical tumor cells had a high N/C ratio and occur singly or in clusters, some of which comprised cells with foamy cytoplasm. Immunohistochemically, tumor cells were positive for CK7 (Fig. 1c) and carcinoembryonic antigen, establishing the diagnosis of EMPD with invasive adenocarcinoma. Tumor cells were positive for PTHrP (Fig. 1d). In contrast, specimens taken from patients with EMPD without hypercalcemia were negative for PTHrP in the tumor cells (data not shown).

\section{Karger'}




\section{Case Reports in Oncology}

\begin{tabular}{l|l}
\hline Case Rep Oncol 2020;13:1209-1214 \\
\hline DOI: 10.1159/000510442 & $\begin{array}{l}\text { @ 2020 The Author(s). Published by S. Karger AG, Basel } \\
\text { www.karger.com/cro }\end{array}$ \\
\hline
\end{tabular}
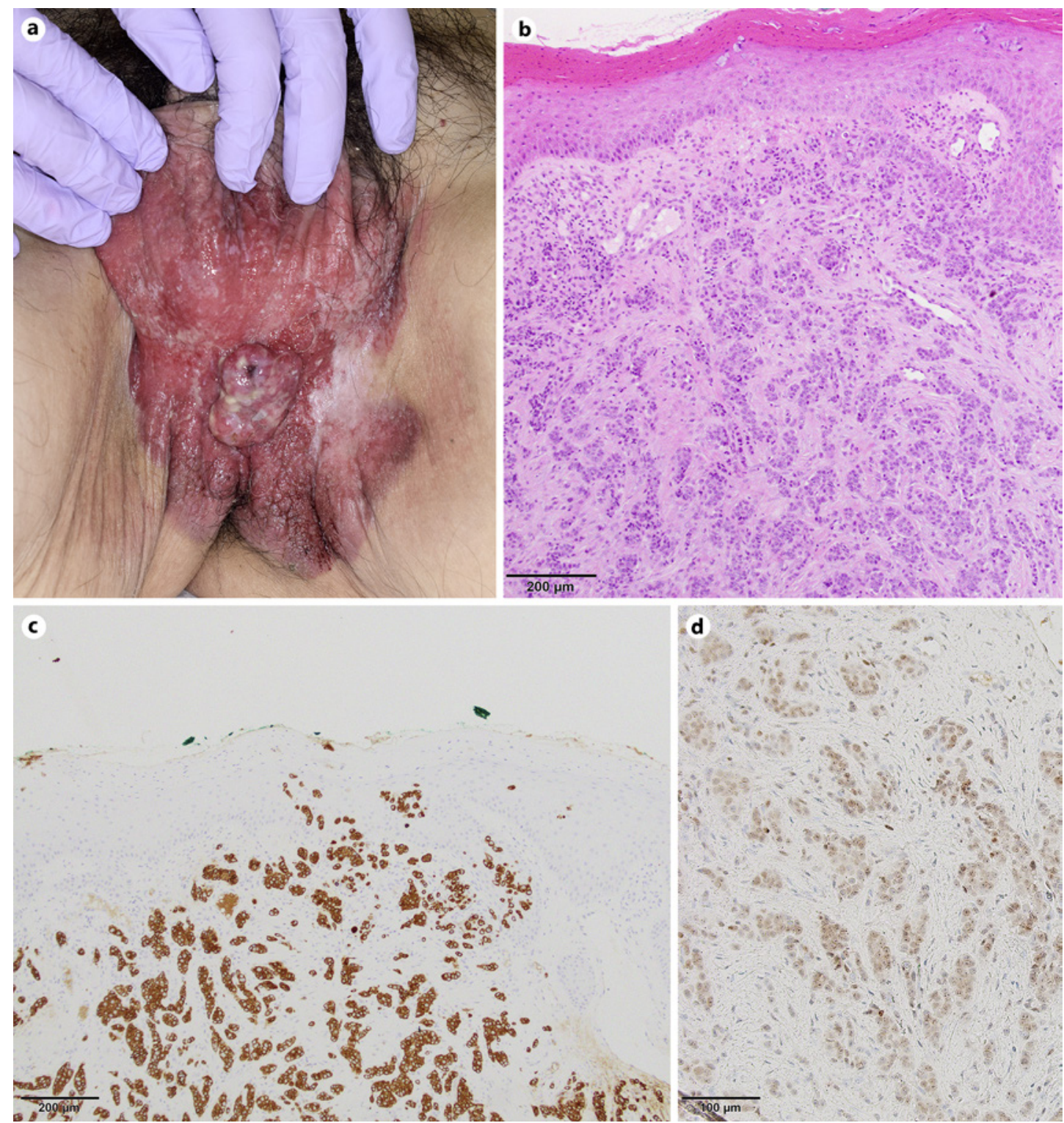

Fig. 1. An eroded, erythematous plaque with a white-coated tumor on the scrotum and perineum (a). The tumor showed infiltration of small, atypical cells arranged in nests and strands in the dermis (b). Positive CK7 (c) and PTHrP (d) staining in tumor cells. Original magnification: $\times 40$ (b); $\times 100$ (c); $\times 200$ (d).

Initial treatment for hypercalcemia with saline, $20 \mathrm{mg} /$ day of furosemide, and $3 \mathrm{mg} /$ day of zoledronic acid hydrate reduced the level of serum calcium within the reference value. As a result, the level of consciousness was also slightly improved. Chemotherapy could not be performed due to a poor performance status (PS4). Radiation therapy with $30 \mathrm{~Gy} / 10 \mathrm{Fr}$ electron beam was effective to reduce the exudate and tumor size (Fig. 2a). However, hypercalcemia developed again on day 18 and the level of serum-corrected calcium elevated up to $18.2 \mathrm{mg} / \mathrm{dL}$, in spite of all the treatment with 40 units of elcatonin, $40 \mathrm{mg}$ of furosemide, and zoledronic acid. Concurrently, the liver metastasis was rapidly enlarged (Fig. 2b), and his general condition gradually deteriorated. The patient died on day 55. Figure 3 summarizes the clinical course. 


\section{Case Reports in Oncology} www.karger.com/cro

Sato et al.: Hypercalcemia in Extramammary Paget's Disease
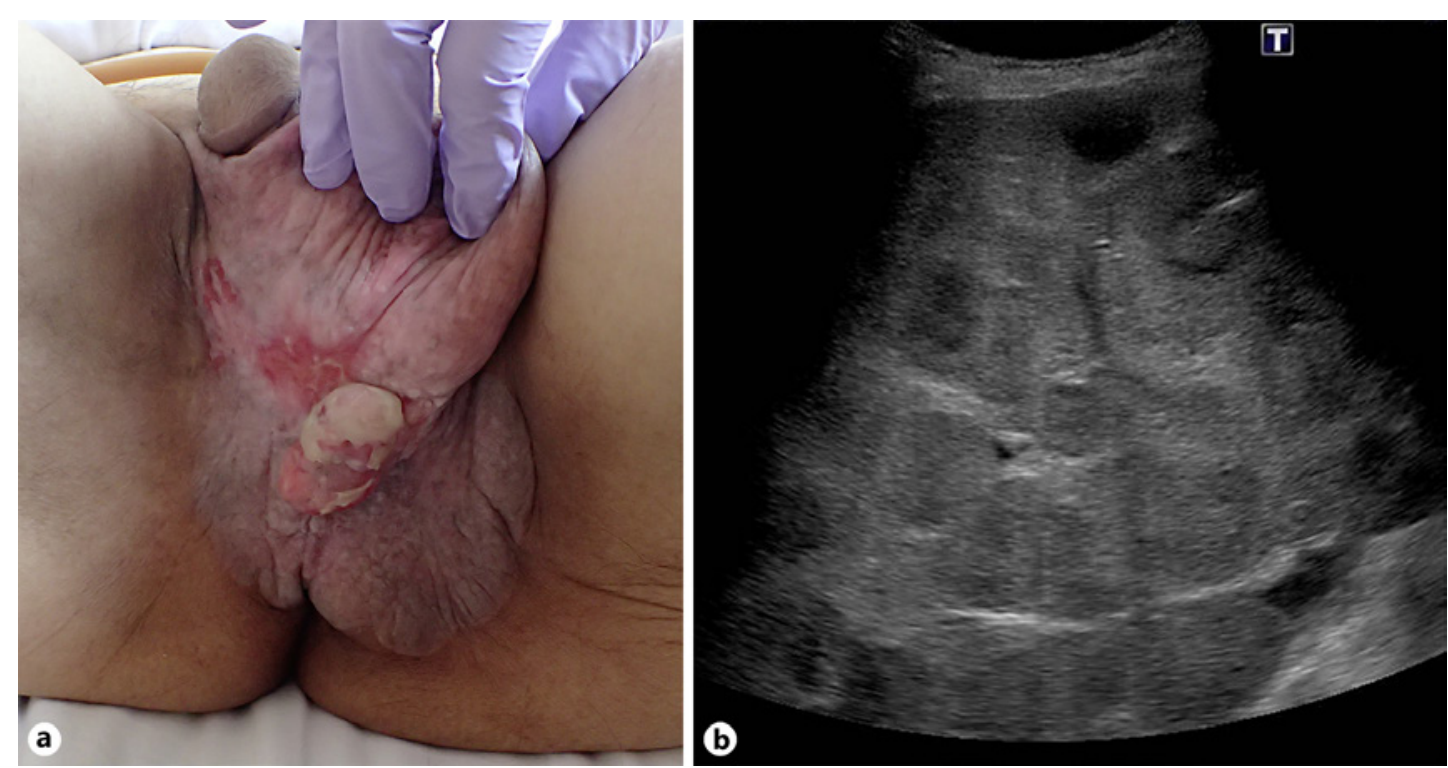

Fig. 2. Partial response observed on radiotherapy (a). The exudate was reduced and tumor size was decreased. Ultrasound image (b); multiple liver metastases were significantly enlarged.

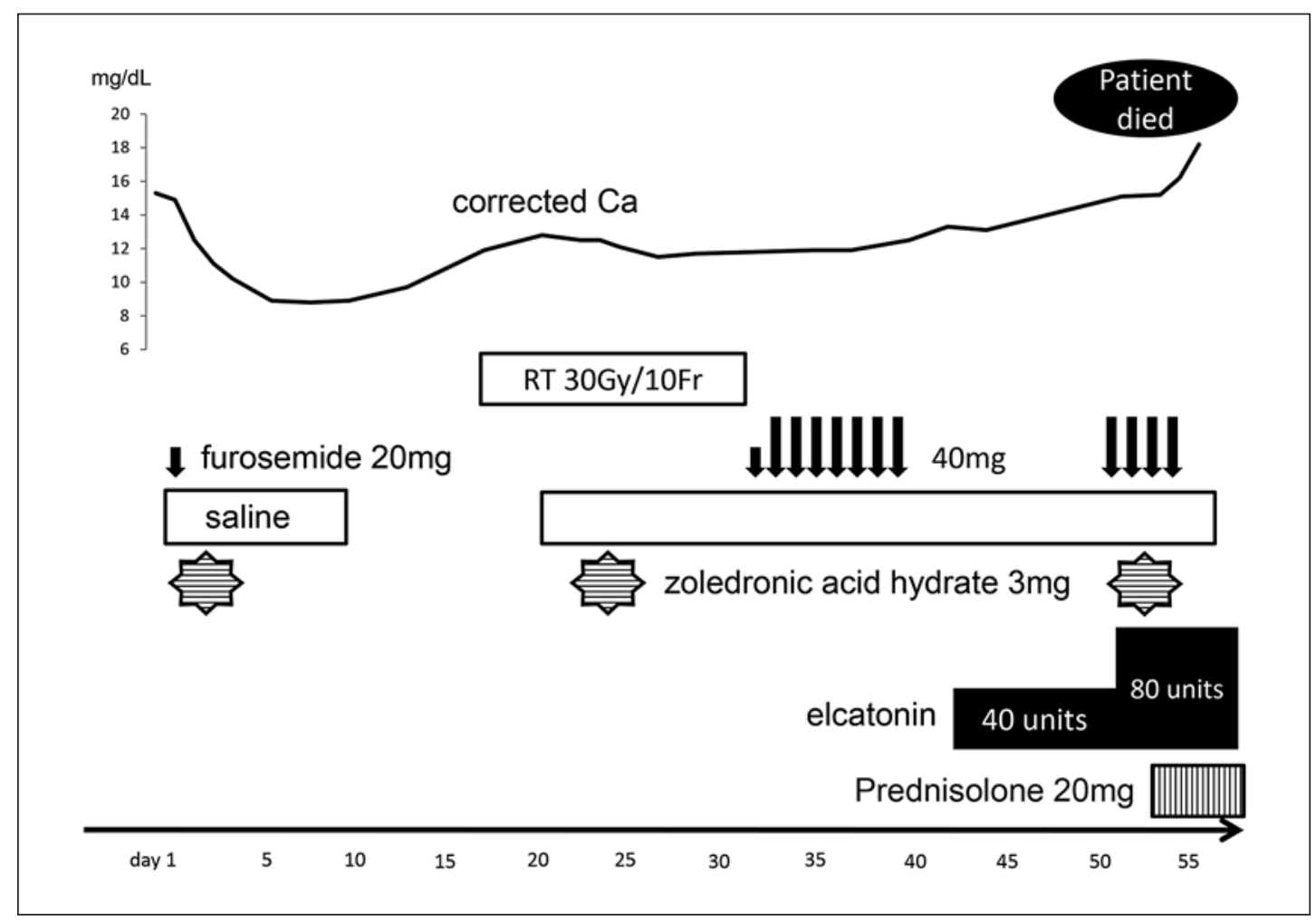

Fig. 3. Summary of the clinical course. 
Sato et al.: Hypercalcemia in Extramammary Paget's Disease

\section{Discussion}

Hypercalcemia has been reported to occur in $20-44 \%$ of patients with cancer [1-3]. Among them, hypercalcemia caused by PTHrP is the most common with $80 \%$. The other proposed mechanisms for hypercalcemia associated with malignancies include: local osteolytic hypercalcemia with secretion of other humoral factors responsible for hypercalcemia; excess extrarenal activated vitamin $\mathrm{D}\left(1,25(\mathrm{OH})_{2}\right.$ vitamin $\left.\mathrm{D}_{3}\right)$; ectopic or primary PTH secretion [1-3]. The elevated serum-intact PTHrP level, the suppressed serum PTH level, and the expression of PTHrP in the tumor cells indicated that HHM due to secretion of PTHrP by Paget cells caused hypercalcemia in the present case.

HHM has been reported in squamous cell carcinoma (e.g., of the head and neck, esophagus, cervix, or lung) and renal cancer among others [1]. Analyses of cancer patients in the UK show that the most common HHM cancers are lung cancer, multiple myeloma, and renal cell carcinoma, followed by breast and colorectal cancers [5]. PTHrP producing EMPD has not been reported in English language literature. Two cases of PTHrP producing EMPD have been reported in Japanese literature, both of which describe male patients presenting plaque and/or tumor, liver metastasis, and positive staining for PTHrP in tumor cells $[6,7]$.

PTHrP is structurally similar to PTH and, like PTH, it enhances renal tubular reabsorption of calcium while simultaneously increasing urinary phosphorus excretion. PTHrP acts on osteoblasts, leading to enhanced synthesis of receptor activator of nuclear factor kappa B ligand (RANKL) which binds to RANK surface receptor on the osteoclast and promotes bone break down (resorption) [2, 3]. The two-site PTHrP immunoradiometric assays (IRMAs) are appropriate for detecting biologically active PTHrP, because they detect full-length amino acids and are sensitive to as low as $0.1-1.0 \mathrm{pmol} / \mathrm{L}$. In the clinical study of 115 patients with cancer, the range of PTHrP examined by IRMAs was 1.2-89.2 pmol/L [8]. A high level of PTHrP-intact, $66.1 \mathrm{pmol} / \mathrm{L}$ by IRMAs, in the present case was assumed to be derived from PTHrP produced by Paget's cells metastatic to the liver, and para-aortic, pelvic, and inguinal lymph nodes as reported previously [6, 7].

Although there are no guidelines available regarding the management of hypercalcemia of malignancy, therapy should be aimed at inhibiting bone resorption and promoting renal calcium excretion $[2,3]$. Bisphosphonates induce osteoclast apoptosis, and reduce osteoclastic bone resorption through indirect mechanisms. Calcitonin lowers blood calcium level mainly by inhibiting osteoclast activity in bones. In the present case, the initial treatment with hydration and zoledronic acid was effective to reduce serum calcium to a normal level with improvement of behavioral problems. However, 2nd and 3rd treatment for relapse of hypercalcemia combined with elcatonin failed to correct the calcium level. The effectiveness of RANKL antibody, denosumab, has been reported in such cases of hypercalcemia of malignancy $[9,10]$. By binding to RANKL to prevent ligand interaction with RANK receptors on precursor osteoclasts, denosumab interferes with osteoclast maturation, function and survival [10]. Moreover, recent studies revealed that Paget's cells profoundly express RANKL and RANKL-RANK interaction between Paget's cells and CD163+Arg1+M2 macrophage plays a key role in establishing an immunosuppressive microenvironment in invasive EMPD [4]. Taken together, the RANKL antibody might be useful not only for patients with humoral hypercalcemia of malignancy but also effective for preventing invasive EMPD. It is of interest to see whether such dual effectiveness is sustained as additional patients with invasive EMPD presenting hypercalcemia are treated with denosumab.

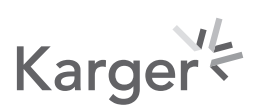




\section{Case Reports in Oncology}

Case Rep Oncol 2020;13:1209-1214

\begin{tabular}{l|l}
\hline DOI: $10.1159 / 000510442$ & c 2020 The Author(s). Published by S. Karger AG, Basel
\end{tabular} www.karger.com/cro

Sato et al.: Hypercalcemia in Extramammary Paget's Disease

\section{Statement of Ethics}

We obtained written informed consent from the patient's son to publish this case including publication of images.

\section{Conflict of Interest Statement}

All authors declare no potential conflicts of interest related to the publication of this case report.

\section{Funding Sources}

We declare that the authors received no funds for this research.

\section{Author Contributions}

T.S. reported the case and wrote the manuscript. T.S., Y.W., N.K., T.Y., W.F., and R.T. were involved in the treatment of this patient. T.Y., Y.A., W.F., and R.T. participated in critically revising the manuscript. All authors approved the final submitted version.

\section{References}

1 Stewart AF. Clinical practice. Hypercalcemia associated with cancer. N Engl J Med. 2005 Jan;352(4):373-9

2 Mirrakhimov AE. Hypercalcemia of malignancy: An update on pathogenesis and management. N Am J Med Sci. 2015 Nov; 7(11):483-93.

3 Goldner W, More S. Treating cancer-related hypercalcemia with gallium nitrate. J Support Oncol. 2016 May; 2(6):509-16.

4 Fukuda K, Funakoshi T. Metastatic extramammary Paget's disease: pathogenesis and novel therapeutic approach. Front Oncol. 2018 Feb;8:38

5 Jick S, Li L, Gastanaga VM, Liede A. Prevalence of hypercalcemia of malignancy among cancer patients in the UK: Analysis of the Clinical Practice Research Datalink database. Cancer Epidemiol. 2015 Dec; 39(6):901-7.

6 Sakamoto M, Koh S, Tatsuoka Y, Katsuyama E, Katakami H. A case of PTHrP producing extramammary Paget's disease. Horm Rinsho. 1997 Nov;45(11 Suppl l):131-3.

7 Hirano K, Uchiyama T, Yamaguchi Y, Okonogi H, Ikeda M, Yokoyama K, et al. A case of PTHrP producing extramammary Paget's disease. J Jpn Soc Dial Ther. 2008 May;41(Suppl 1):780.

8 Jin J, Chung JO, Chung MY, Cho DH, Chung DJ. Clinical characteristics, causes and survival in 115 cancer patients with parathyroid hormone related protein-mediated hypercalcemia. J Bone Metab. 2017 Nov; 24(4):249-55.

9 Ashihara N, Nakajima K, Nakamura Y, Kobayashi M, Shirahata K, Maeda C, et al. Denosumab is effective for controlling serum calcium levels in patients with humoral hypercalcemia of malignancy syndrome: a case report on parathyroid hormone-related protein-producing cholangiocarcinoma. Intern Med, 2016 Dec; 55(23):3453-7.

10 Dietzek A, Connelly K, Cotugno M, Bartel S, McDonnell AM. Denosumab in hypercalcemia of malignancy: a case series. J Oncol Pharm Pract. 2015 Apr;21(2):143-7.

\section{Karger'}

